\title{
Focused transhepatic electroporation mediated by hypersaline infusion through the portal vein in rat model. Preliminary results on differential conductivity
}

\author{
Clara Pañella1, Quim Castellví2, Xavier Moll ${ }^{3}$, Rita Quesada ${ }^{1}$, Alberto Villanueva ${ }^{4}$, \\ Mar Iglesias ${ }^{5}$, Dolores Naranjo ${ }^{5}$, Patricia Sánchez-Velázquez ${ }^{1}$, Anna Andaluz ${ }^{3}$, Luís Grande ${ }^{1}$, \\ Antoni Ivorra ${ }^{2}$ and Fernando Burdío ${ }^{1}$
}

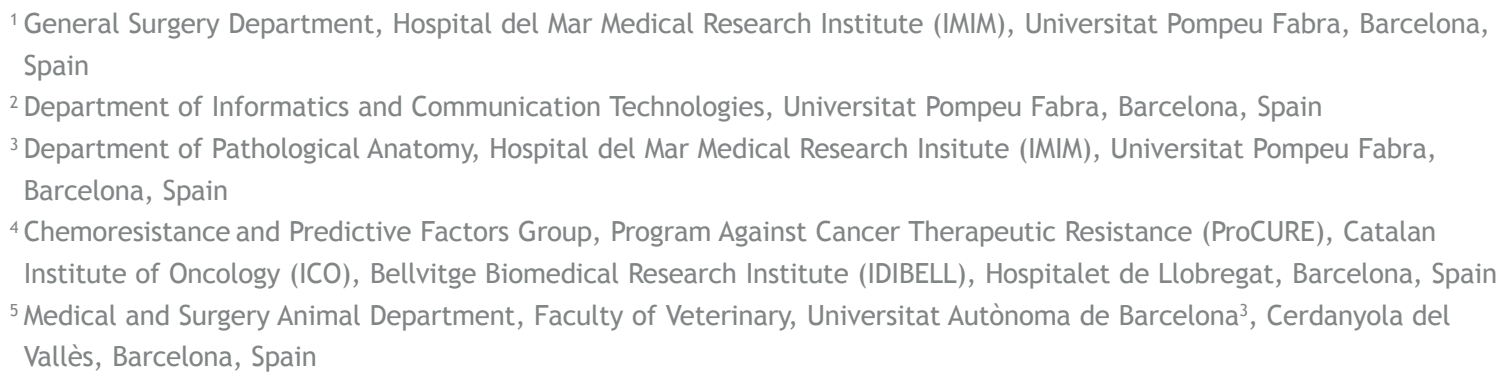

Radiol Oncol 2017; 51(4): 415-421.

Received 2 July 2017

Accepted 22 October 2017

Correspondence to: Fernando Burdío M.D., Clara Pañella M.D., Unidad de Cirugía Hepática. Servicio de Cirugía General y Digestiva, Hospital del Mar de Barcelona, Passeig Maritim 25-29, 08003 Barcelona, Spain. E-mail: fburdio@hotmail.com, cpvilamu@gmail.com

Disclosure: No potential conflicts of interest were disclosed.

Background. Spread hepatic tumours are not suitable for treatment either by surgery or conventional ablation methods. The aim of this study was to evaluate feasibility and safety of selectively increasing the healthy hepatic conductivity by the hypersaline infusion (HI) through the portal vein. We hypothesize this will allow simultaneous safe treatment of all nodules by irreversible electroporation (IRE) when applied in a transhepatic fashion.

Material and methods. Sprague Dawley (Group A, $n=10$ ) and Athymic rats with implanted hepatic tumour (Group $\mathrm{B}, \mathrm{n}=8$ ) were employed. $\mathrm{HI}$ was performed ( $\mathrm{NaCl} 20 \%, 3.8 \mathrm{~mL} / \mathrm{Kg}$ ) by trans-splenic puncture. Deionized serum ( $40 \mathrm{~mL} /$ $\mathrm{Kg}$ ) and furosemide $(2 \mathrm{~mL} / \mathrm{Kg}$ ) were simultaneously infused through the jugular vein to compensate hypernatremia. Changes in conductivity were monitored in the hepatic and tumour tissue. The period in which hepatic conductivity was higher than tumour conductivity was defined as the therapeutic window (TW). Animals were monitored during 1-month follow-up. The animals were sacrificed and selective samples were used for histological analysis.

Results. The overall survival rate was $82.4 \%$ after the $\mathrm{HI}$ protocol. The mean maximum hepatic conductivity after $\mathrm{HI}$ was 2.7 and 3.5 times higher than the baseline value, in group A and B, respectively. The mean maximum hepatic conductivity after HI was 1.4 times higher than tumour tissue in group B creating a TW to implement selective IRE.

Conclusions. HI through the portal vein is safe when the hypersaline overload is compensated with deionized serum and it may provide a TW for focused IRE treatment on tumour nodules.

Key words: irreversible electroporation; liver tumour; electrical conductivity

\section{Introduction}

Electroporation (EP) is the phenomenon by which the cell membrane permeability is increased when the cell is subjected to high electric fields. Such increase in membrane permeability can irreversibly alter cell homeostasis and lead to cell death, either by necrosis or apoptosis. In this case the term irre- 
versible electroporation (IRE) is used..$^{1-5}$ IRE is typically performed by inserting thin needle electrodes into the targeted tissue and delivering a number (8-100) of short (10-100 $\mu$ s) high-voltages pulses across the electrodes, thereby producing field magnitudes in the tissue in the order of $1000 \mathrm{~V} / \mathrm{cm}$ or more. $^{3}$

The ideal ablation system for multiple hepatic nodules should treat the tumoural tissue while preserving the remaining healthy hepatic tissue. Unfortunately, the IRE as it is used today, does not allow the preservation of the surrounding healthy tissue. Although, it is a non-thermal technique and is credited with sparing the extracellular matrix, IRE leads to the apoptosis of either healthy or tumoural hepatic tissue where the electric field is applied. ${ }^{5-10}$ In fact, this technique is highly dependent on tissue conductivity ${ }^{11-13}$ and tumours have higher dielectric properties (and higher conductivities) than normal healthy tissue because of the higher sodium and water content of cancer cells. ${ }^{14}$ This higher conductivity may lead to a lower electric field in the tumoural tissue than in the surrounding healthy tissue. ${ }^{12}$

(A)

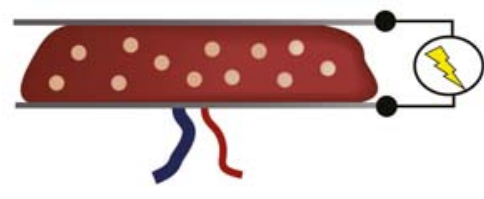

Without hypersaline infusion through the portal vein

(C)
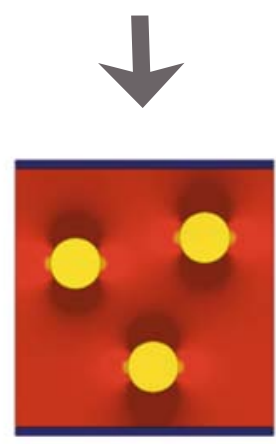

Electric Field (V/cm)

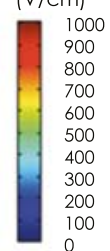

$\sigma_{\text {liver }}=0.12 \mathrm{~s} / \mathrm{m}$
(B)

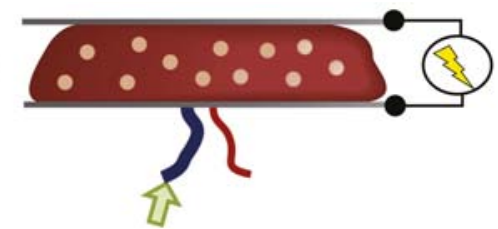

With hypersaline infusion through the portal vein ( $\mathrm{NaCl} 20 \%$ )

(D)

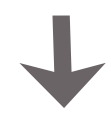

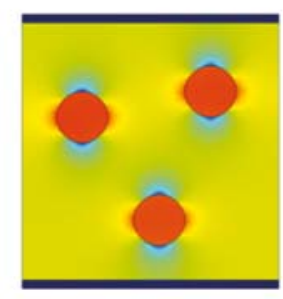

$\sigma_{\text {liver }}=1.2 \mathrm{~s} / \mathrm{m}$
Electric Field $(\mathrm{V} / \mathrm{cm})$

1000 900 800
700 600 500 400 300 200 100
FIGURE 1. IRE application model with plate electrodes, in scattered tumoural liver without (A) or with (B) hypersaline infusion through the portal vein and its corresponding numerical simulation ( $C$ and $D$ ) obtained from the received electric field in healthy and tumoural tissue - own results obtained from our previous research $(17,18)$. (C) Without hypersaline infusion through the portal vein, there is not selective effect on scattered tumoural nodules. Nevertheless, in (D) with hypersaline infusion through the portal vein, there is an increase on the electric field on scattered tumours (meaning a preferential ablative IRE effect on these nodules) because of the increase in healthy tissue conductivity $(\sigma)$.
Our group recently studied the long-term effectiveness of treating a large amount of healthy hepatic tissue harbouring an implanted tumour with parallel transhepatic electrodes. ${ }^{15}$ In this study we showed that even with high electrical fields (up to $2000 \mathrm{~V} / \mathrm{cm}$ ) complete eradication of the tumour was infrequent even when massive destruction of nearby healthy hepatic tissue was present (expressed by life-threating ionic imbalances). ${ }^{16}$

In this context, we recently used a mathematical model aiming to assess the electrical conductivity of the liver after introducing hypertonic solution $(20 \% \mathrm{NaCl})$ through the portal vasculature (Figure 1). This model was further validated with an in vivo pilot safety study on pigs, which demonstrated that this infusion could increase the mean basal conductivity by up to 4 times. ${ }^{17}$ Interestingly, the tumour nodules lack of sinusoids means they are only supplied with blood from the hepatic artery. ${ }^{18-20}$ In fact, this characteristic is currently used for identifying tumours by injecting contrast agents with CT or MRI. Hypersaline infusion through the portal vein would therefore raise the electrical conductivity of the healthy hepatic tissue, so that, when a potential difference is applied between the opposite sides of the liver, the electrical field magnitude in the tumours would be significantly larger than in the rest of the healthy tissue. Thus it would make possible to produce electroporation in tumour cells and without affecting healthy hepatic tissue (Figure 1). ${ }^{18}$

The aim of this study was to evaluate feasibility and safety of selectively increasing of healthy hepatic conductivity by HI through the portal vein as a means to increase the electric field in scattered tumoural nodules with transhepatic IRE.

\section{Materials and methods}

All aspects of this study had the approval of the Ethics Committee on Animal Research of the Government of Catalonia (FBP-13-1474P2 procedure, DAAM: 7016). This animal experimental study was conducted following directives 2010/63/ EU of the European Parliament and Council of 22 September 2010, for the protection of animals for experimental and scientific purposes.

\section{Animal model}

Two animal models were considered in the study: Group A $(\mathrm{n}=10)$ Sprague Dawley rats (Charles River Laboratories, Kingston, NY, USA) and Group 
B (n = 8) Athymic nude rats Hsd:RH-Foxn $1^{\mathrm{mu}}$ (Harlan laboratories, Indianapolis, IN, USA). Eighteen six-week-old males were used and all of them were maintained under standard conditions with a laboratory animals diet and water ad libitum. We supervised all the animals following Morton and Griffiths' guidelines on the recognition of pain, distress and discomfort in experimental animals. ${ }^{21}$

Group A was used to develop the HI protocol and show that it had no influence on survival rate; and group B was conceived for tumour implantation and conductivity test on changes between hepatic and tumour tissue.

\section{Tumour implantation technique}

Primary colorectal metastases were obtained from Bellvitge Hospital (HUB) and the Catalan Institute of Oncology (ICO) with approval by the Ethical Committee, and ethical and legal protection guidelines of human subjects, including informed consent, were followed. Primary metastasis was implanted orthotopically in the liver of Crl:NUFOXn1 nu mice, and when the orthoxenograft or patient derived orthotopic xenograft (PDOX) growth, the mice were sacrificed. Then, the tumour was minced and re-implanted in the left hepatic lobe of group B, after a quarantine period at the

(A)

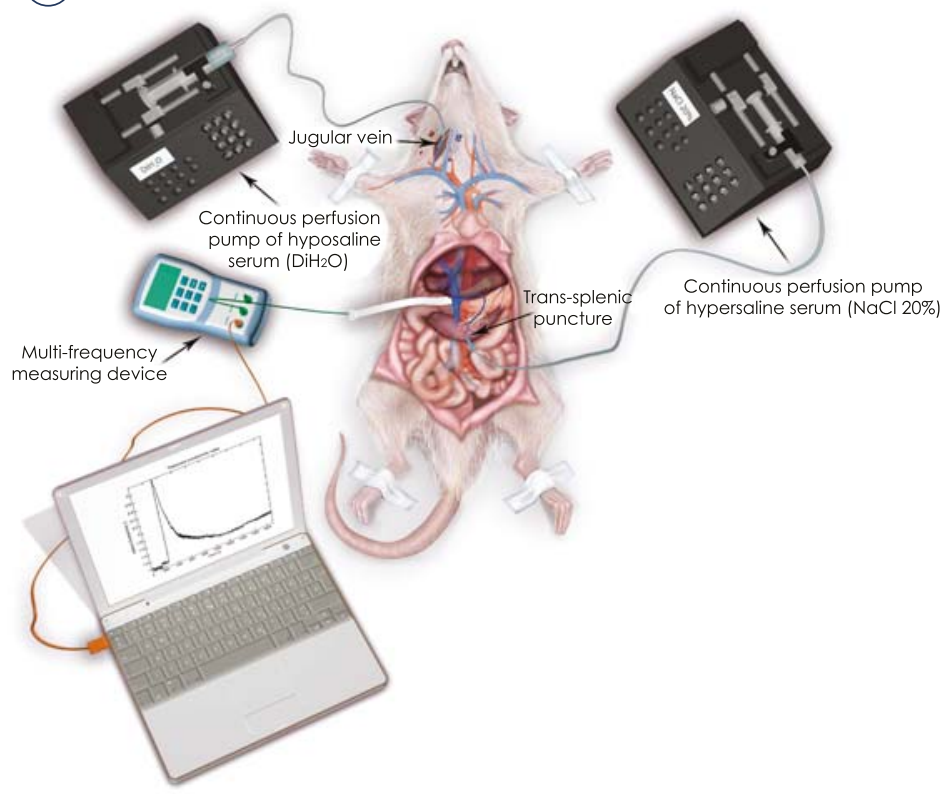

age of six weeks, by midline laparotomy under general anaesthesia (mixture of isofluorane and inhaled oxygen). Tumour pieces of measuring 5-8 $\mathrm{mm}^{2}$ were sutured with non-reabsorbable prolene $6 / 0$ on the surface of the left hepatic lobe. The midline laparotomy closure was made up of a running suture within the muscle layer with reabsorbable monocryl 5/0 and skin with simple silk 5/0 stitches. The tumour grew for a mean of 20 days to allow adequate hepatic implantation. All the animals developed a tumour on the left hepatic lobe, appropriate for conductivity measurement after HI through the portal vein.

\section{Hypersaline infusion protocol}

HI was applied by means of a hypersaline infusion at $20 \%(\mathrm{NaCl})$, which is an aqueous solution with high conductivity (204 S/m). ${ }^{22,23}$

Once anaesthetised by inhalation anaesthesia (isoflurane $4 \%$ for induction and $2.5 \%$ for maintenance with $\mathrm{O}_{2} 0.8 \mathrm{~L} /$ minute), subcutaneous analgesia was administered (buprenorphine $0.05 \mathrm{mg} /$ $\mathrm{Kg}$ ). The abdomen and anterior cervical area were shaved and prepared aseptically with iodine. The liver was exposed by iterative abdominal incision and dressing pad underneath, after 7 days after the tumour implantation in group A and after mean of

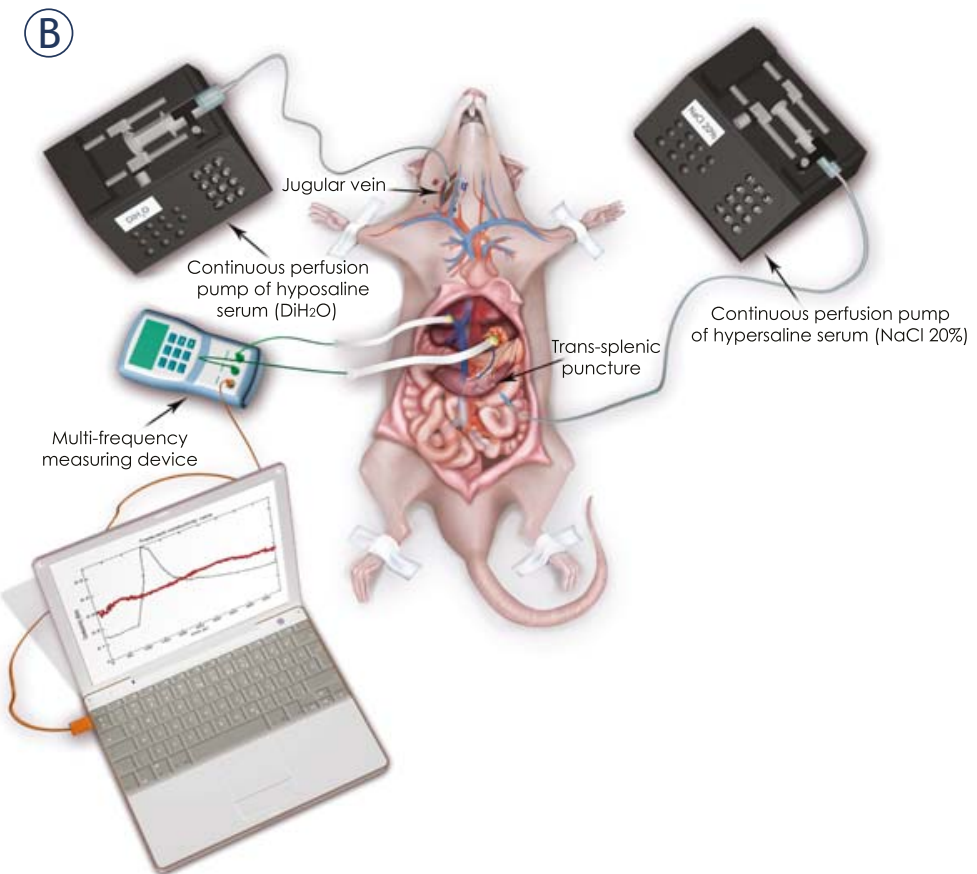

FIGURE 2. General setting of the hypersaline infusion protocol through the portal vein in Sprague Dawley rats (A) and in Athimic rats with implanted tumour (B). After exposing spleen at abdominal midline, hypersaline infusion was performed by trans-splenic puncture with a pump. Hyposaline infusion through the jugular vein was performed to compensate the systemic hypernatremia. Two tetrapolar setup electrodes measured tumour and healthy hepatic conductivity and data were collected with a multifrecuency device and gathered in a computer. 
20 days of tumour growth in group B. The anterior vertical cervicotomy approach allowed anterior jugular vein dissection and catheterization with a flexible plastic tube $\left(\right.$ Tygon $^{\circledR}$ ND 100-80 tubing, USP corporation, USA) for deionised serum infusion $\left(\mathrm{DiH}_{2} \mathrm{O}\right)$. The infusion of hyposaline serum, $\mathrm{DiH}_{2} \mathrm{O},(40 \mathrm{~mL} / \mathrm{Kg})^{18}$, through the anterior jugular vein, avoids the HI side effects, such as systemic hypernatremia. Prior to the HI protocol, the animals were pre-hydrated with a quarter of the total hyposaline serum $\left(\mathrm{DiH}_{2} \mathrm{O}\right)$, calculated by weight, at the same infusion rate as the $\mathrm{HI}$. $\mathrm{NaCl} 20 \%$ (3.8 $\mathrm{mL} / \mathrm{Kg}$ ) was then injected into the portal vein by trans-splenic puncture $\left(27 \mathrm{G}\right.$ needle) ${ }^{18}$, during hyposaline infusion. The HI rate was set up to administer the dose in 1 minute. Trans-splenic puncture was used for the HI protocol, since splenic vein is a part of the portomesenteric system and infusion is more feasible than dissection of the portal vein (Figure 2).

Intravenous infusion of furosemide $(2 \mathrm{~mL} / \mathrm{Kg})$ was administered before and after HI protocol in order to compensate the fluid overload after intravenous administration of hyposaline serum (via jugular) and hypersaline serum (via trans-splenic). ${ }^{18}$

\section{Electrical conductivity measurement}

Hepatic and tumour conductivity were measured using the tetrapolar method following the setup employed in ${ }^{24}$. This consists of four stainless steel needles $(0.25 \mathrm{~mm}$ of diameter and $3.5 \mathrm{~mm}$ of exposure) placed in a straight line with $0.5 \mathrm{~mm}$ between the centres of the needles. Tumour electrode was inserted at the centre of the tumour, with fourneedle shape, in a way that it favoured its stability.
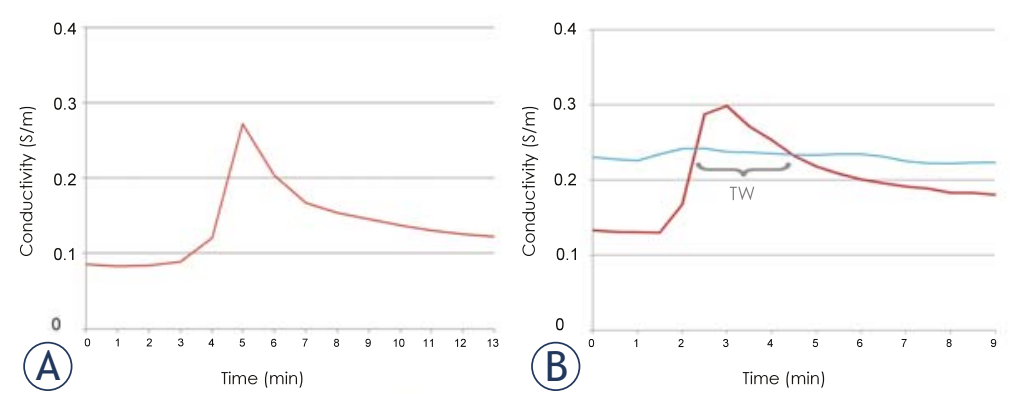

- Tumour conductivity $(\mathrm{S} / \mathrm{m})$

- Liver conductivity $(\mathrm{S} / \mathrm{m})$

FIGURE 3. Conductivity changes after hypersaline infusion protocol in a representative case of group A (Sprague Dawley) and B (Athimic rats with implanted tumour). In both groups, a sharp increase and a posterior slowing decrease of conductivity in healthy hepatic tissue was observed. On the contrary, conductivity of the tumoural tissue (Group B) was stable. Thus, it created a therapeutic window (TW), the safety period where IRE it would be applied.
Measurements were performed at ten-excitation frequencies from $5 \mathrm{kHz}$ to $1 \mathrm{MHz}$ at a rate of one sweep per second. Signal generation and the acquisition of both current and voltage across the sample were performed by a Red Pitaya board and a front-end..$^{25}$

\section{Post-surgical management}

During the surgical procedure and during the immediate postoperative period, a heat lamp or heat pad controlled the risk of hypothermia. Administered analgesia was buprenorphine 0.05 $\mathrm{mg} / \mathrm{Kg}$ s.c. and meloxicam $1 \mathrm{mg} / \mathrm{Kg}$ s.c., both every 24 hours for 3 days. An oral prophylactic antibiotic was also supplied: enrofloxacin in drinking water, $500 \mathrm{mg} / \mathrm{Kg}$, for the first 3 days. Weight was monitored periodically as an objective sign of positive outcome after catheterization and the HI protocol.

\section{Histopathological samples}

Animals were sacrificed according to modified Morton and Griffiths criteria ${ }^{21}$ if signs of suffering or distress were observed. Animals of group A were sacrificed 1 month after the procedure while animals of group B were sacrificed in case of clinical evidence of tumour dissemination (for instance, subcutaneous tumour implants). At necropsy, liver and spleen were collected from both groups. Tissue samples were fixed on formaldehyde and kept in paraffin blocks. Sections of $3 \mu \mathrm{m}$ were prepared for routine haematoxylin-eosin staining (HEOS) slides.

\section{Statistical analyses}

All the statistics were processed by the SPSS statistical software package (SPSS, version 21, IBM, Armonk, NY, USA) and expressed as mean \pm standard deviation. Since the sample was small, the Wilcoxon test was used for non-parametric data to make pairwise comparisons of weight and conductivity of healthy tissue before and after HI, in both groups. A P-value of $<0.05$ was considered statistically significant.

\section{Results}

\section{Hypersaline infusion through the portal vein is safe when compensated}

The HI protocol was reproduced in $94.4 \%$ (17/18) of cases in group A and B. One animal died from anaesthetic problems before the HI protocol. Overall 
TABLE 1. Comparison between two groups A (Sprague Dawley) and B (Athimic). *PO: Postoperative

\begin{tabular}{lcccc} 
& GROUP A & p-value & GROUP B & p-value \\
\hline Administration of HI protocol & \multicolumn{2}{c}{$\mathrm{n}=9$} & & $\mathrm{n}=8$ \\
Weight increase during PO* (grams) & $100 \pm 30.57$ & 0.012 & $-1.94 \pm 37.81$ & 0.386 \\
Baseline Conductivity in healthy tissue $(\mathrm{S} / \mathrm{m})$ & $0.10 \pm 0.02$ & & $0.13 \pm 0.02$ & 0.008 \\
Conductivity after HI in healthy tissue $(\mathrm{S} / \mathrm{m})$ & $0.27 \pm 0.75$ & 0.008 & $0.49 \pm 0.17$ & $0.24 \pm 0.03$ \\
Baseline Conductivity in tumour tissue $(\mathrm{S} / \mathrm{m})$ & & $\mathrm{NA}$ & & $175 \pm 115$ \\
Therapeutic window $(\mathrm{sec})$ & & $\mathrm{NA}$ & & \\
\hline
\end{tabular}

technical-related survival rate was $82.4 \%(14 / 17)$ after the $\mathrm{HI}$ protocol for both groups and there were 3 technical-related deaths (one animal in group A and 2 in group B). In all of these technical-related deaths, extra HI was administered after observing recurrent reflux of the HI through the transsplenic puncture and no correct compensation of the HI overload was possible in these cases. Two died shortly after the HI protocol and the other was sacrificed two days after when it was seen to be lethargic (see comments on this animal in histology section). During the postoperative period of the animals with HI protocol, a mean increase of weight of 100 grams was observed in the other animals in group A (from $366.5 \pm 51.6$ grams to $466.5 \pm 71.9$ grams at the necropsy date).

\section{Implantation and tumour development}

The polyclonal human colon adenocarcinoma tumour model was well implanted with a correct growth after 60-90 days and with no evidence of peritoneal carcinomatosis at necropsy in all cases. Mean tumour volume, at implantation, was $122.5 \pm 84.3 \mathrm{~mm}^{3}$ and it remained stable at the time of the HI protocol, being $121 \pm 65.11 \mathrm{~mm}^{3}$. Mean tumour volume at the necropsy was $692.8 \pm 347.3$ $\mathrm{mm}^{3}$.

As expected in this tumoural model, the mean weight of the animals in group B initially increased after tumour implantation (from $158 \pm 7.33$ grams to a mean maximum weight of $217.34 \pm 9.19$ grams), but then decreased $(156.06 \pm 39.68$ grams, at necropsy) (Table 1 ).

\section{Hypersaline infusion increases conductivity in healthy hepatic parenchyma}

In all animals increased hepatic tissue conductivity was observed after the HI protocol. In group A,
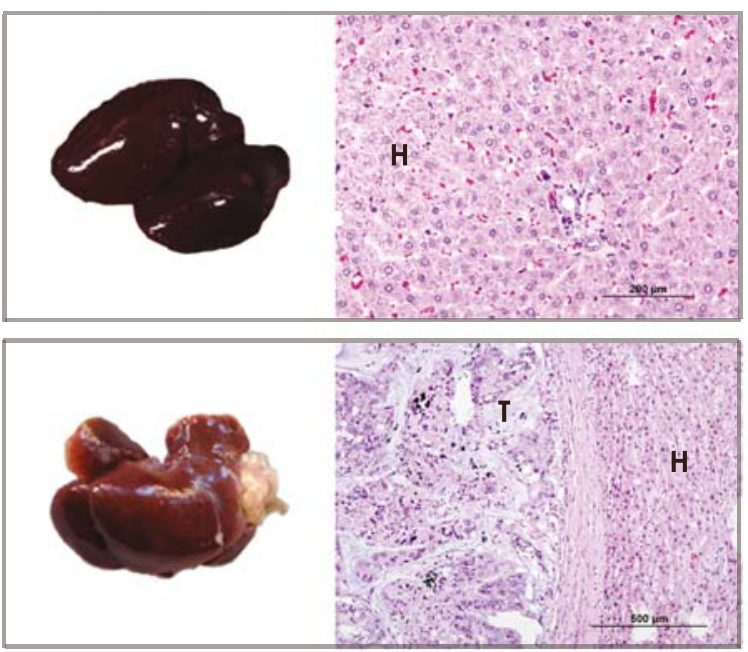

FIGURE 4. These were representative livers from group A (Sprague Dawley) and group B (Athimic rats with implanted tumour) after $\mathrm{HI}$ protocol at the 4th week of postoperative. Histological analysis revealed the indemnity of the architecture and morphology of healthy hepatocytes $(\mathrm{H})$ of both groups A and B. In group B, histological samples showed that $\mathrm{HI}$ protocol did not interfere in tumour (T) evolution.

the mean baseline conductivity before administering $\mathrm{HI}$ was $0.10 \pm 0.02 \mathrm{~S} / \mathrm{m}$ and the mean maximum conductivity peaked at $0.27 \pm 0.75 \mathrm{~S} / \mathrm{m}$. Likewise, in group $\mathrm{B}$, the mean baseline hepatic conductivity was $0.11 \pm 0.02 \mathrm{~S} / \mathrm{m}$ and the mean maximum hepatic conductivity after $\mathrm{HI}$ was $0.38 \pm 0.16 \mathrm{~S} / \mathrm{m}$ (range 0.19-0.66 S/m), which was around 3.5 times higher than the baseline value (Figure 3).

\section{Hypersaline infusion through the portal vein does not change tumoural conductivity}

As expected, tumoural conductivity in group B was not influenced by HI (mean value: $0.24 \pm 0.03$ $\mathrm{S} / \mathrm{m})$. The higher conductivity in healthy hepatic tissue created a mean TW of $175 \pm 115.23$ seconds (Figure 3B), where the mean maximum hepatic 
conductivity after $\mathrm{HI}$ was 1.4 times higher (range 1.04-1.76) than tumour tissue. In this TW, electroporation could therefore be advantageously applied.

\section{Histopathological evaluation}

In group $\mathrm{A}$, hepatic parenchyma exhibited normal architecture, with polygonal hepatocytes and preserved porta trials (Figure 4A). Congestion in vessels was seldom scattered throughout healthy hepatic tissue. No changes were present in cellular component or architecture after HI.

Similar results to group A were found in group $B$, with no architectural or morphological damage after the HI protocol. However, the animal that was sacrificed two days after two extra doses of HI showed patchy areas of coagulation necrosis in the liver especially around the central veins of acini. No damage in neoplastic tissue was observed (Figure 4B).

\section{Discussion}

The preliminary results show that the HI employed in this study selectively increases the conductivity of healthy tissue, so that when the electric pulses are applied to a whole hepatic section, the magnitude of the electric field in the tumours would be significantly higher than in the rest of the healthy tissue. The results also show that this infusion could be safe when it is correctly compensated as we previously demonstrated in the pilot study on pigs. ${ }^{17}$

Concerning the safety of the protocol, it should be remembered that hypersaline solutions has been extensively used to resuscitate patients with traumatic brain injury. ${ }^{26}$ It is currently assumed that the maximum dosage at $20 \%$ is $1.8 \mathrm{ml} / \mathrm{Kg}$. Twice this dose was used in the present study (3.8 $\mathrm{ml} / \mathrm{Kg}$ ) combined with measures to compensate the corresponding hypernatremia (doses of hyposaline serum through the anterior jugular vein and furosemide). With this protocol all the animals survived the HI protocol, with the appropriate increase in their body weight and no histological damage being found at necropsy. The three animal deaths were related to extra HI doses (administered after observing recurrent reflux of the infusion through the trans-splenic puncture) without correct compensation for the overload.

As regards the potential translation of these conductivity results, the basal hepatic and tu- moural conductivity observed are similar to those described in the literature for humans and in the tumour itself can vary up to $20 \% .{ }^{27}$ For example, Laufer et al. found $0.12 \mathrm{~S} / \mathrm{m}$ and $0.27 \mathrm{~S} / \mathrm{m}$ for normal and tumoural liver tissue, respectively. ${ }^{24}$ Although little data is available in the literature on increasing conductivity by administering $\mathrm{HI}$ through the portal vein, in our previous study on pigs, with the same HI protocol, similar peak conductivity results were obtained (range 0.21-0.37 S/m) ${ }^{17}$, which further supports the consistency of the present data despite the small sample size. Finally, our actual TW $(175 \pm 115.2 \mathrm{sec})$ appears to be enough to implement a conventional IRE protocol. Although the application protocols are varied, most studies use between 70 and 90 pulses for duration of 70-100 $\mu \mathrm{s} .{ }^{28-30}$ For instance, our previous IRE protocol with plate electrodes lasted for 100 seconds (90-100 pulses at a frequency of $1 \mathrm{~Hz}){ }^{16,31}$ Our protocol IRE lasted 100 seconds, without pauses. However, pauses can be reduced in case the TW values obtained in this preliminary study are set in this range of time, in future essays with bigger sample size. In other words, the IRE protocol can be adjusted according to the average TW obtained.

In conclusion, the presented preliminary results provide evidence in favour of safety of administering HI through the portal vein when compensated and show that it may provide a therapeutic window for focused IRE treatment on tumour nodules. Further studies should be carried out to confirm these results, giving that this study is a preliminary research.

\section{Acknowledgements}

This work received financial support from the Spanish Government (Ministry of Economy and Competitiveness) under Grant TEC2014-52383-C3. The authors disclose no conflicts of interests.

\section{References}

1. Appelbaum L, Ben-David E, Faroja M, Nissenbaum Y, Sosna J, Goldberg SN. Irreversible electroporation ablation: creation of large-volume ablation zones in in vivo porcine liver with four-electrode arrays. Radiology 2014; 270: 416-24. doi: 10.1148/radiol.13130349

2. Charpentier KP, Wolf F, Noble L, Winn B, Resnick M, Dupuy DE. Irreversible electroporation of the liver and liver hilum in swine. HPB (Oxford) 2011; 13: 168-73. doi: 10.1111/j.1477-2574.2010.00261.x

3. Davalos RV, Mir IL, Rubinsky B. Tissue ablation with irreversible electroporation. Ann Biomed Eng 2005; 33: 223-31. doi: 10.1111/j.14772574.2010.00261.x 
4. Bower M, Sherwood L, Li Y, Martin R. Irreversible electroporation of the pancreas: definitive local therapy without systemic effects. J Surg Oncol 2011; 104: 22-8. doi: 10.1002/jso.21899

5. Daniels C, Rubinsky B. Electrical field and temperature model of nonthermal irreversible electroporation in heterogeneous tissues. J Biomech Eng 2009; 131: 071006. doi: 10.1115/1.3156808

6. Al-Sakere B, Bernat C, Andre F, Connault E, Opolon P, Davalos RV, et al. A study of the immunological response to tumor ablation with irreversible electroporation. Technol Cancer Res Treat 2007; 6: 301-6. doi: $10.1177 / 153303460700600406$

7. Bellard E, Markelc B, Pelofy S, Le Guerroue F, Sersa G, Teissie J, et al. Intravital microscopy at the single vessel level brings new insights of vascular modification mechanisms induced by electropermeabilization. I Control Release 2012; 163: 396-403. doi: 10.1016/j.jconrel.2012.09.010

8. Dollinger M, Zeman F, Niessen C, Lang SA, Beyer LP, Muller M, et al. Bile duct injury after irreversible electroporation of hepatic malignancies: evaluation of MR imaging findings and laboratory values. J Vasc Interv Radiol 2016; 27: 96-103. doi: 10.1016/j.jvir.2015.10.002

9. Kanthou C, Kranjc S, Sersa G, Tozer G, Zupanic A, Cemazar M. The endothelial cytoskeleton as a target of electroporation-based therapies. Mol Cancer Ther 2006; 5: 3145-52. doi: 10.1158/1535-7163.MCT-06-0410

10. Maor E, Rubinsky B. Endovascular nonthermal irreversible electroporation: a finite element analysis. J Biomech Eng 2010; 132: 031008. doi: 10.1115/1.4001035

11. Ben-David E, Ahmed M, Faroja M, Moussa M, Wandel A, Sosna J, et al. Irreversible electroporation: treatment effect is susceptible to local environment and tissue properties. Radiology 2013; 269: 738-47. doi: 10.1148/ radiol.13122590

12. Ivorra A, Villemejane J, Mir LM. Electrical modeling of the influence of medium conductivity on electroporation. Phys Chem Chem Phys 2010; 12: 10055-64. doi: 10.1089/c004419a

13. Qasrawi R, Silve L, Burdio F, Abdeen Z, Ivorra A. Anatomically realistic simulations of liver ablation by irreversible electroporation. Technol Cancer Res Treat 2017. doi: 10.1177/1533034616687477. [Epub ahead of print]

14. Peyman A, Kos B, Djokic M, Trotovsek B, Limbaeck-Stokin C, Sersa G, et al. Variation in dielectric properties due to pathological changes in human liver. Bioelectromagnetics 2015; 36: 603-12. doi: 10.1002/bem.21939

15. Sanchez-Velazquez P, Castellvi Q, Villanueva A, Iglesias $M$, Quesada R, Panella $C$, et al. Long-term effectiveness of irreversible electroporation in a murine model of colorectal liver metastasis. Sci Rep 2017; 7: 44821. doi: $10.1038 /$ srep44821

16. Sanchez-Velazquez P, Castellvi Q, Villanueva A, Quesada R, Panella C, Caceres $\mathrm{M}$, et al. Irreversible electroporation of the liver: is there a safe limit to the ablation volume? Sci Rep 2016; 6: 23781. doi: 10.1038/srep23781

17. Castellvi $Q$, Sanchez-Velazquez $P$, Moll X, Berjano E, Andaluz A, Burdio F, et al. Modeling liver electrical conductivity during hypertonic injection. Int $J$ Numer Meth Bio 2017. doi: 10.1002/cnm.2904. [Epub ahead of print]

18. Castellví $Q$, Sánchez-Velázquez $P$, Berjano E, Burdío F, Ivorra A. Selective electroporation of liver tumor nodules by means of hypersaline infusion: A feasibility study. In: Lacković I, Vasic D, editors. 6th European Conference of the International Federation for Medical and Biological Engineering: MBEC 2014, 7-11 September 2014, Dubrovnik, Croatia. Cham: Springer International Publishing; 2015. p. 821-4. doi: 10.1007/978-3-319-111285_204

19. Dezso K, Bugyik E, Papp V, Laszlo V, Dome B, Tovari J, et al. Development of arterial blood supply in experimental liver metastases. Am J Pathol 2009; 175: 835-43. doi: 10.2353/ajpath.2009.090095

20. Liu Y, Matsui O. Changes of intratumoral microvessels and blood perfusion during establishment of hepatic metastases in mice. Radiology 2007; 243: 386-95. doi: 10.1148/radiol.2432060341

21. Morton DB, Griffiths PH. Guidelines on the recognition of pain, distress and discomfort in experimental animals and an hypothesis for assessment. Vet $\operatorname{Rec} 1985$; 116: 431-6.

22. Sala J, Guardia E, Marti J. Effects of concentration on structure, dielectric, and dynamic properties of aqueous $\mathrm{NaCl}$ solutions using a polarizable model. J Chem Phys 2010; 132: 214505. doi: 10.1063/1.3429253
23. Yamaguchi T, Matsuoka T, Koda S. A theoretical study on the frequency-dependent electric conductivity of electrolyte solutions. II. Effect of hydrodynamic interaction. J Chem Phys 2009; 130: 094506. doi: 10-1063/1.3085717

24. Laufer S, Ivorra A, Reuter VE, Rubinsky B, Solomon SB. Electrical impedance characterization of normal and cancerous human hepatic tissue. Physiol Meas 2010; 31: 995-1009. doi: 10.1088/0967-3334/31/7/009

25. Ruiz-Vargas A, Arkwright JW, Ivorra A, editors. A portable bioimpedance measurement system based on Red Pitaya for monitoring and detecting abnormalities in the gastrointestinal tract. 2016 IEEE EMBS Conference on Biomedical Engineering and Sciences (IECBES); 2016 4-8 Dec. 2016. doi: 10.1016/j.hpb.2016.03.609

26. Tyagi R, Donaldson $\mathrm{K}$, Loftus $\mathrm{CM}$, Jallo J. Hypertonic saline: a clinical review. Neurosurg Rev 2007; 30: 277-89; discussion 89-90. doi: 10.1007/s10143007-0091-7

27. Muftuler LT, Hamamura MJ, Birgul O, Nalcioglu O. In vivo MRI electrical impedance tomography (MREIT) of tumors. Technol Cancer Res Treat 2006; 5: 381-7.

28. Valerio M, Dickinson L, Ali A, Ramachandran N, Donaldson I, Freeman A, et al. A prospective development study investigating focal irreversible electroporation in men with localised prostate cancer: Nanoknife Electroporation Ablation Trial (NEAT). Contemp Clin Trials 2014; 39: 57-65. doi: 10.1016/j.cct.2014.07.006

29. van den Bos W, de Bruin DM, Muller BG, Varkarakis IM, Karagiannis AA, Zondervan PJ, et al. The safety and efficacy of irreversible electroporation for the ablation of prostate cancer: a multicentre prospective human in vivo pilot study protocol. BMJ open 2014; 4: e006382. doi: 10.1136/bmjopen-2014-006382

30. Wendler JJ, Porsch M, Nitschke S, Kollermann J, Siedentopf S, Pech M, et al. A prospective Phase 2a pilot study investigating focal percutaneous irreversible electroporation (IRE) ablation by NanoKnife in patients with localised renal cell carcinoma (RCC) with delayed interval tumour resection (IRENE trial). Contemp Clin Trials 2015; 43: 10-9. doi: 10.1016/j.cct.2015.05.002

31. Sahakian AV, Al-Angari HM, Adeyanju OO. Electrode activation sequencing employing conductivity changes in irreversible electroporation tissue ablation. IEEE Trans Biomed Eng 2012; 59: 604-7. doi: 10.1109/ TBME.2011.2180722 